\title{
Some conjugate WP-Bailey pairs and transformation formulas for $q$-series
}

\author{
H. M. SRivastava ${ }^{1,2}$, S. N. Singh ${ }^{3}$, S. P. Singh ${ }^{3}$ and Vijay YAdAV ${ }^{4}$
}

\section{ABSTRACT.}

In this paper, the authors prove several theorems involving $q$-series identities by applying a certain family of conjugate WP-Bailey pairs. Making use of these theorems in conjunction with some WP-Bailey pairs, various transformation formulas for $q$-series are also established.

Acknowledgements. The second-named author (S. N. Singh) is thankful to the Department of Science and Technology of the Government of India (New Delhi, India) for support under a major research project No. SR/S4/MS:735/2011 dated 07 May 2013, entitled "A Study of Transformation Theory of q-Series, Modular Equations, Continued Fractions and Ramanujan's Mock-Theta Functions," under which this work was initiated.

\section{REFERENCES}

[1] Andrews, G. E., Bailey's transform, lemma, chains and tree, in Special Functions 2000 : Current Perspective and Future Directions, (Proceedings of the NATO Advanced Study Institute; Tempe, Arizona, May 29June 9, 2000), NATO Sci. Ser. II Math. Phys. Chem., Vol. 30, Kluwer Academic Publishers, Dordrecht, Boston and London, 2001, pp. 1-22

[2] Bailey, W. N., Identities of the Rogers-Ramanujan type, Proc. London Math Soc., (Ser. 2). 50 (1949), 1-10

[3] Cao, J. and Srivastava,H. M., Some q-generating functions of the Carlitz and Srivastava-Agarwal types associated with the generalized Hahn polynomials and the generalized Rogers-Szegö polynomials, Appl. Math. Comput., 219 (2013), 8398-8406

[4] Choi, J. and Srivastava, H. M., $q$-Extensions of a multivariable and multiparameter generalization of the Gottlieb polynomials in several variables, Tokyo J. Math., 37 (2014), 111-125

[5] Luo, Q.-M. and Srivastava, H. M., q-Extensions of some relationships between the Bernoulli and Euler polynomials, Taiwanese J. Math., 15 (2011), 241-257

[6] Gasper, G. and Rahman, M., Basic Hypergeometric Series (with a Foreword by Richard Askey), Encyclopedia of Mathematics and Its Applications, Vol. 35, Cambridge University Press, Cambridge, New York, Port Chester, Melbourne and Sydney, 1990; Second edition, Encyclopedia of Mathematics and Its Applications, Vol. 96, Cambridge University Press, Cambridge, London and New York, 2004

[7] McLaughlin, J., Sills, A. V. and Zimmer, P., Lifting Bailey pairs to WP-Bailey pairs, Discrete Math., 309 (2009), 5077-5091

[8] Srivastava, H. M., Some generalizations and basic (or q-) extensions of the Bernoulli, Euler and Genocchi polynomials, Appl. Math. Inform. Sci., 5 (2011), 390-444

[9] Srivastava, H. M. and Choi, J., Zeta and q-Zeta Functions and Associated Series and Integrals, Elsevier Science Publishers, Amsterdam, London and New York, 2012

[10] Srivastava, H. M. and Karlsson, P. W., Multiple Gaussian Hypergeometric Series, Halsted Press (Ellis Horwood Limited, Chichester), John Wiley and Sons, New York, Chichester, Brisbane and Toronto, 1985

[11] Verma, A., On identities of Rogers-Ramanujan type, Indian J. Pure Appl. Math., 11 (1980), 770-790

[12] Verma, A. and Jain, V. K., Certain summation formulas for q-series, J. Indian Math. Soc. (New Ser.), 47 (1983), 71-85

1 Department of Mathematics and Statistics

UNIVERSITY OF VICTORIA

VICTORIA, BRITISH COLUMBIA V8W 3R4, CANADA

2 China Medical University

TAichung 40402, TAiWAN, Republic of China

E-mail address: harimsri@math.uvic.ca

${ }^{3}$ Department of Mathematics

Tilak Dhari Post-Graduate College

JAUNPUR 222002, UTTAR PRADESH, INDIA

E-mail address: snsp39@gmail.com, snsp39@yahoo.com

${ }^{4}$ Department of Mathematics and Statistics

S. P. D. T. COLLEGE

ANDHERI (EAST), Mumbai 400059, MAHARASHTRA, INDiA

E-mail address: vijaychottu@yahoo.com 